\title{
Archéopages
}

Archéopages

Archéologie et société

$36 \mid 01 / 2013$

Exotismes

\section{Les lampes plastiques et le luminaire à tête de Nubien du mithraeum d'Angers}

\section{Maxime Mortreau}

\section{(2) OpenEdition}

1 Journals

Édition électronique

URL : https://journals.openedition.org/archeopages/206

DOI : 10.4000/archeopages.206

ISSN : 2269-9872

Éditeur

INRAP - Institut national de recherches archéologiques préventives

Édition imprimée

Date de publication : 1 novembre 2013

Pagination : 16-17

ISSN : 1622-8545

\section{Référence électronique}

Maxime Mortreau, "Les lampes plastiques et le luminaire à tête de Nubien du mithraeum d'Angers ", Archéopages [En ligne], 36 | 01/2013, mis en ligne le 01 janvier 2015, consulté le 23 janvier 2022. URL http://journals.openedition.org/archeopages/206 ; DOI : https://doi.org/10.4000/archeopages.206 


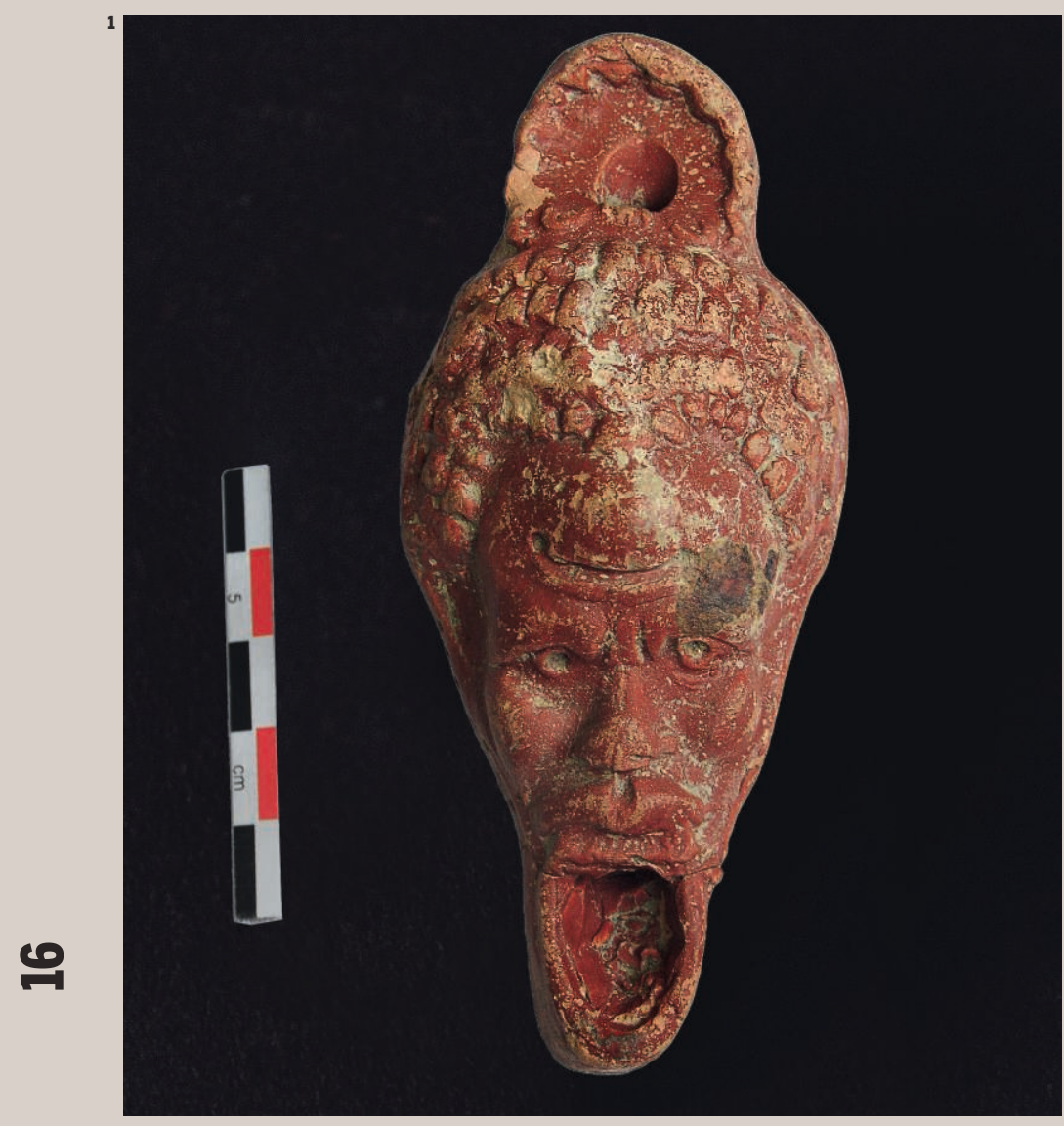

Ies lampes plastiques et le luminaire à tête de Nubien du mithraeum d'Angers

Maxime Mortreau Inrap, $U_{M R} 6566$ CREAAH

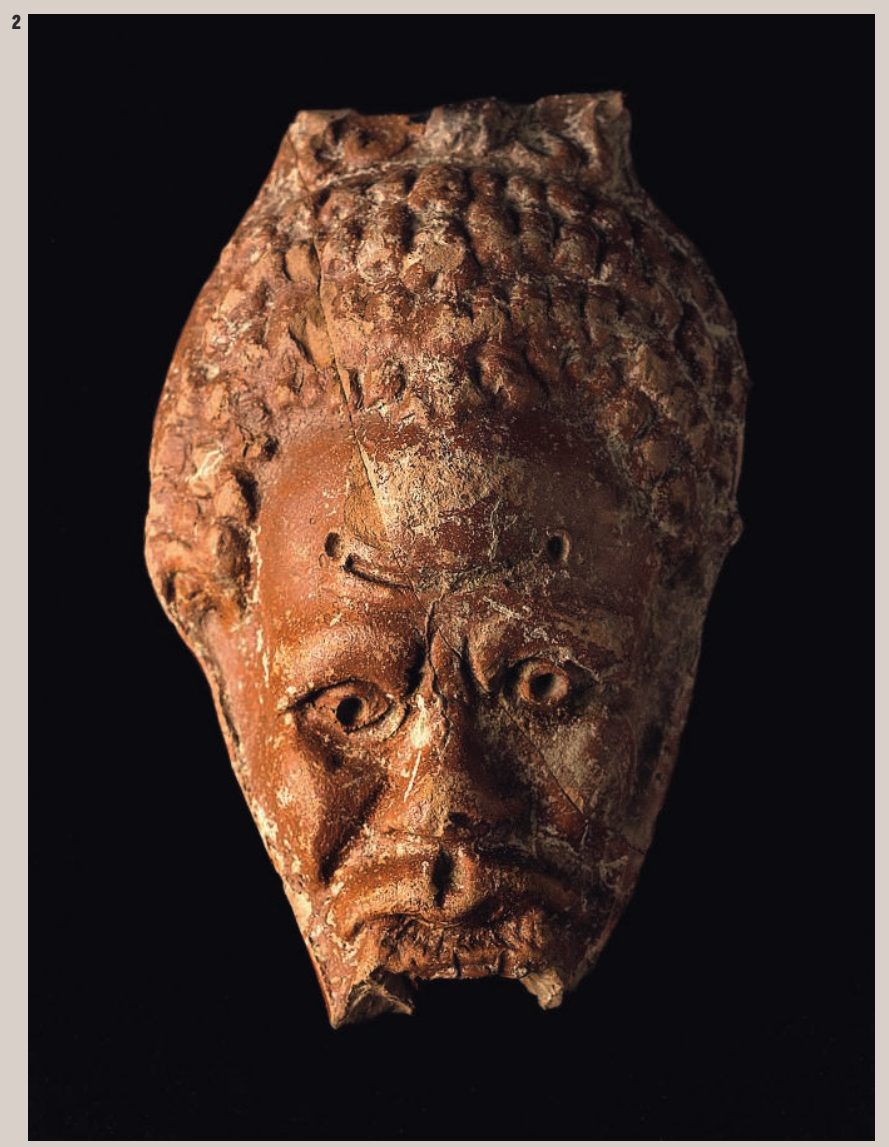

1. Une lampe complète (longueur : 10,01 cm, largeur : $4,35 \mathrm{~cm}$, hauteur : 4,05 cm), réalisée par moulage en deux parties. Une ouverture circulaire a été pratiquée dans la partie supérieure de la tête pour le remplissage.
Les oreilles sont peu visibles, 2. Une des têtes de Nubien cachées au contact des deux du luminaire. parties moulées par un

bourrelet de collage souligne par une série de pointillés estampés. Un dispositif de préhension est fixé à la partic sommitale de la tête.
De nombreux fragments de lampes à tête de Nubien ont été recueillis dans les niveaux de destruction/démolition du mithraeum d'Angers. Ils ont permis de reconstituer cinq lampes simples [ill. 1] et un luminaire formé de 15 lampes [ill. 2 et 3 ]. Dans chaque cas, la figure est celle d'un Noir africain (un Nubien ou un itthiopien?) aux caractéristiques physiques accentuées : nez épaté, bouche charnue, pommettes saillantes, arcades sourcilières épaisses, front souligné par un pli convexe, cheveux coiffés en tresses, menton prognathe. En choisissant ce type de représentation pour les luminaires de leur lieu de culte, les fondateurs du mithraeum avaient sans doute une intention particulière. Les Africains, en dehors de leur condition d'esclave, de soldat ou de gladiateur, avaient-ils un rôle particulier dans le culte de Mithra ? On sait qu'ils tenaient une place importante dans les cérémonies du culte à mystères d'Isis en tant que prêtres, musiciens ou danseurs (voir le relief funéraire du $\mathrm{Ir}^{\mathrm{e}}$ siècle en marbre provenant d'Arricia et conservé à Rome au Museo Nazionale Romano). Mais il semblerait aussi que la figure de l'Africain ait eu un pouvoir apotropaïque dans la croyance populaire (Snowden, 1976). Tant pour les lampes simples que pour le luminaire, l'aspect de la pâte et du vernis de la sigillée permet d'identifier une fabrication typique de la période de production de la phase 7 des ateliers de Lezoux (160-230). Pour les lampes, cette attribution est confirmée par l'examen d'une reproduction photographique de la valve supérieure d'un moule de lampe plastique provenant de la collection ollier, attribuée aux ateliers de Lezoux et figurant une tête d'Africain en tout point identique à celles découvertes à Angers (Vertet, 1983, p. 130, $n^{\circ}$ Z). Quant au lustre, il est un exemple unique à l'heure actuelle. Ce type de lampe plastique semble dériver d'un prototype connu dans le bassin méditerranéen, et plus précisément au Maghreb (région où il faut sans doute situer l'origine des lampes copiées à Lezoux). À ce jour, on en répertorie plusieurs autres exemplaires : moule signé Damarion provenant de Délos (Déonna, 1908); exemplaires en bronze, dont celui découvert à Zeugma ; deux lampes d'Afrique du Nord conservées dans les musées de Constantine 
3. Restitution du luminaire; 12 fragments ont été recueillis dans les niveaux de destruction/démolition du mithraeum. Le luminaire reprend la même thématique que les lampes, à la différence près que le plan de pose ne présente pas de décor curviligne. La couronne évidée, d'un diamètre estimé à $24,6 \mathrm{~cm}$, est de section rectangulaire $(4,1 \mathrm{~cm}$ sur $1,9 \mathrm{~cm})$. Elle est percée de trous à mi-hauteur dans l'axe des lampes. Celles-ci, assemblées deux à deux sur la couronne, sont surmontées d'un tenon percé d'un orifice permettant à la fois le remplissage et la suspension.

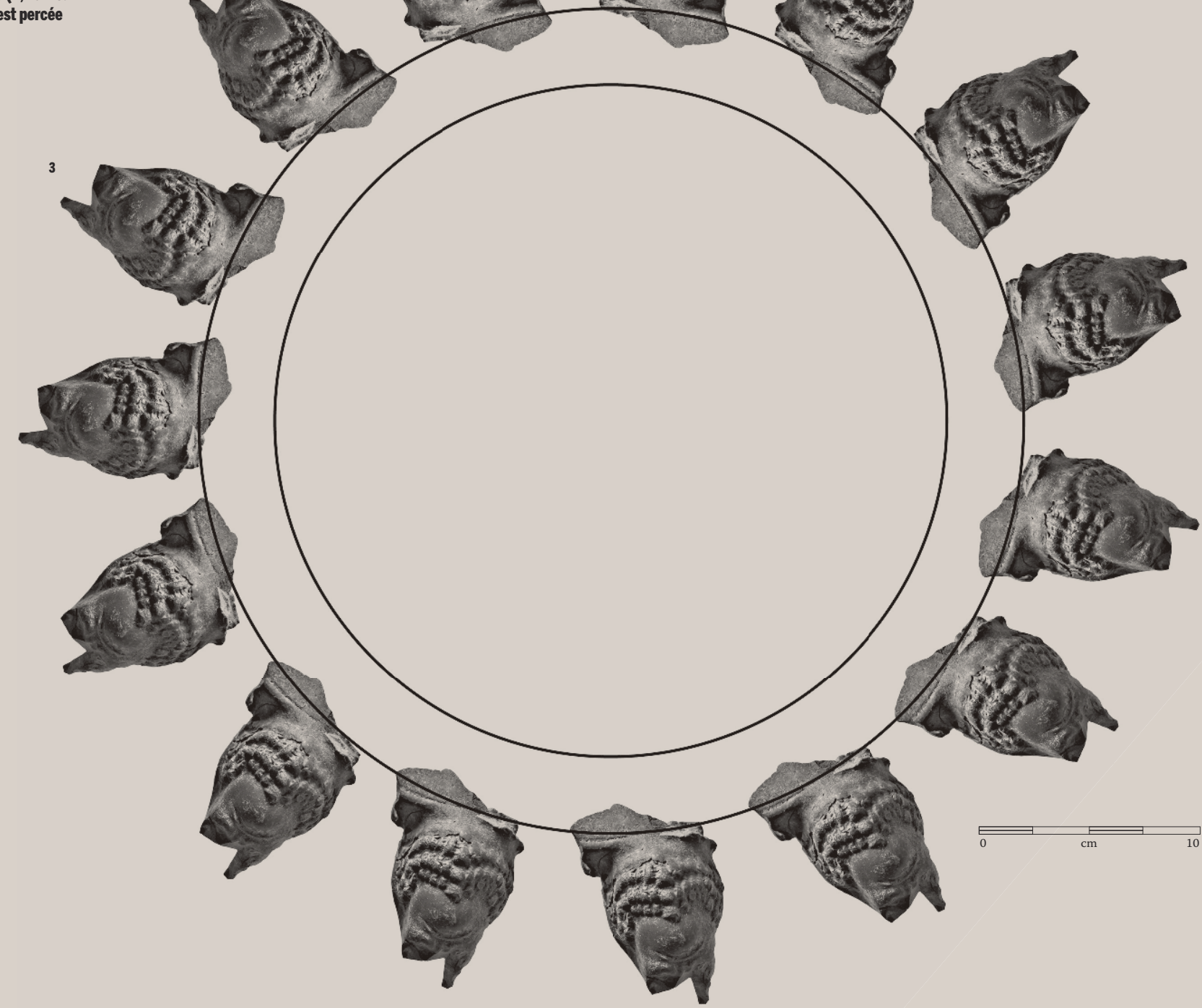

et de Tunis, non datées (Desanges, 1976, p. 261) ; une couronne datant de la fin du I ${ }^{\text {er }}$ siècle, découverte en 2009 dans le Rhône (Martinez (dir.), 2012); un luminaire de la fin $\mathrm{du}_{\mathrm{rv}} \mathrm{e}^{\text {siècle, de }}$ fabrication locale, découvert à Fréjus (Brentchaloff, 2009, p. 282) ; un fragment d'une couronne grossière sur le site du mithraeum de Septeuil en fonction dans la seconde moitié du rve siècle (Barat, Gaidon-Bunuel, Van Ossel, 2006, p. 159,) ; et enfin, un lustre à sept becs de lampe type Firmalampen provenant des fouilles anciennes de Martigny, non loin d'un mithraeum (Chrzanovski (dir.), 2006, p. 57).
Références bibliographiques

BARAT Y GAIDON-BUNUEL M-A. VAN OSSEL P., 2006 : « Les céramiques du mithraeum de Septeuil (Yvelines). Un ensemble du troisième quart du IV ${ }^{\mathrm{e}}$ siècle de notre ère dans la région parisienne ", in Les céramiques de l'Antiquité tardive en Île-de-France et dans le Bassin parisien, vol. I, Ensembles régionaux, Nanterre, ACR (coll. Diocesis Galliarum, Documents de travail, 7), p. 137-16o.

BrentChALOFF D., 2009: « Lampes en terre cuite moulées et modelées », in Goudineau C., BRENTCHAloff D. (DIR.), Le camp de la flotte d'Agrippa à Fréjus. Les fouilles du quartier de Villeneuve (1979-1981), Paris, Errance, p. 277-284.

ChrZANOvSKI L. (DIR.), 2006 : Lumière! L'éclairage dans l'Antiquité, Catalogue d'exposition, Musée romain de Nyon, 15 mai 2003-30 avril 2004, Nyon, Musée romain, 120 p.

DÉONNA W., 1908: « Les lampes antiques de Délos (pl. I-II) », Bulletin de correspondance hellénique, vol. 32, p. 133-176.
DESANGES J., 1976: « L'iconographie du Noir dans l'Afrique du Nord antique », in Vercoutter J., LeClant J., SNOwden F.M., DeSANGes J., L'image du Noir dans l'art occidental, vol. 1, Des pharaons à la chute de l'Empire romain, Fribourg, Office du Livre, p. 246-268.

MARTINEZ J.-L. (DIR.), 2012 : Arles, les fouilles du Rhône. Un fleuve pour mémoire, Arles - Paris, Actes Sud Éditions du musée du Louvre, 48 p.

SNOWDEN F.M., 1976 : « Témoignages iconographiques sur les populations noires dans l'Antiquité grécoromaine », in VERCOUTTER J., LECLANT J., SNOWDEN F.M., Desanges J., L'image du Noir dans l'art occidental, vol. 1, Des pharaons à la chute de l'Empire romain, Fribourg, Office du Livre, p. 133-245.

VERTET H., 1983: Recherches sur les ateliers de potiers de la Gaule centrale, t. III, Les techniques de fabrication des lampes en terre cuite du centre de la Gaule, Le Blanc-Mesnil (coll. Sites, hors-série 20). 